\title{
COVID-19 is shifting the adoption of wearable monitoring and telemedicine (WearTel) in the delivery of healthcare: opinion piece
}

\author{
Ralph J. Mobbs ${ }^{1,2,3}$, Daniel Ho ${ }^{1,2,3}$, Wen Jie Choy ${ }^{1,2,3}$, Callum Betteridge ${ }^{1,2,3}$, Henry Lin $^{4}$ \\ ${ }^{1}$ Faculty of Medicine, University of New South Wales, Sydney, Australia; ${ }^{2}$ NeuroSpine Surgery Research Group (NSURG), Sydney, Australia; \\ ${ }^{3}$ Neurospine Clinic, Prince of Wales Private Hospital, Randwick, NSW 2031, Australia; ${ }^{4}$ Department of Neurosurgery, Prince of Wales Hospital, \\ Randwick, NSW 2031, Australia \\ Correspondence to: Ralph J. Mobbs. Suite 7, Level 7, Prince of Wales Private Hospital, Randwick, NSW 2031, Australia. Email: ralphmobbs@hotmail.com.
}

Submitted May 01, 2020. Accepted for publication Jul 22, 2020.

doi: 10.21037/atm-20-3678

View this article at: http://dx.doi.org/10.21037/atm-20-3678

\section{Introduction}

The novel coronavirus, COVID-19, changed the world within a matter of weeks. The primary action to constrain the spread of the virus is social isolation. Given this public health principle, and the shortage of personal protective equipment during this global pandemic, all healthcare stakeholders need to reconsider the indications for faceto-face healthcare encounters in providing patient care. Many questions have been raised during the escalation of this global pandemic. Which encounters are imperative and which ones can be switched to non-face-to-face care? What changes in laws, regulations, payment policies and workflow are needed to enable this transition (1-3)?

Non-face-to-face (remote) care models where the patient is physically separated from the physician (or other healthcare worker), are empowered by communicationbased technologies such as video conferencing and the use of continuous patient monitoring (wearable or "surface sensor") technologies that capture patient metrics and deliver health data remotely to the physician. These technologies have existed for some time, however widespread implementation has been constrained by outdated laws, regulations and policies. Governments, insurers and commercial payers urgently need to accept that patient care models must change, and that telehealth and wearable monitoring can provide equivalent, or advanced, levels of care. Until recently, evidence for the effectiveness of telehealth and wearable monitoring, compared to traditional care models, has been scarce $(4,5)$.

The combination of Telemedicine as an audiovisual communications platform, and Wearable technologies collecting and transmitting real-time health metrics provides numerous benefits to both health providers and patients alike. The COVID-19 crisis presents a strong argument for all involved in healthcare to transition to remote care models when appropriate. The authors termed the phrase WearTel (Wearables/Telehealth) to describe the transformation of healthcare delivery from the clinic, to remote monitoring and care delivery in the home. In addition, this philosophy potentially frees up hospital resources to those who will generate maximal benefit from face-to-face care and hospitalisation.

\section{Relevant technologies}

\section{Telemedicine}

The general term for many non-face-to-face services include "telemedicine" or "telehealth", from the Greek word of "at a distance". Many thinks of telemedicine as consisting exclusively of real time interactive or synchronous audiovisual communication between a patient and provider. There is a wide spectrum of other telehealth services including telephone communication, asynchronous digital services including email, text messages and provider-to-provider communications (6). Some also extend the definition of telemedicine to include administration and remote provision

$\wedge$ ORCID: 0000-0001-6542-6836 


\author{
Health metric \\ Vital signs ( $\mathrm{HR}, \mathrm{SpO}_{2}, \mathrm{RR}, \mathrm{BP}$, body temp) \\ Sleep patterns \\ General activity levels (activity minutes, daily step count) \\ Gait assessment
}

Falls detection and prediction

Joint function

Blood sugar levels

Electrolyte measurement

\section{Relevant pathology}

All critical conditions

Sleep disorders, mood disorders such as anxiety

Obesity, postoperative monitoring of lower limb and spine surgery

Neurological gait disorders, postoperative monitoring of lower limb and spine surgery

Neurological gait disorders, frailty

Knee, hip and shoulder pathologies and surgery

Type 1 diabetes, other endocrine disorders

Electrolyte disorders, arrhythmias

Figure 1 Health metric $v s$. pathology that would benefit from relevant data acquisition.

of education and professional development (7).

The majority of evaluation and management services can be performed remotely. Even though telehealth lends itself well to conducting outpatient consultations, it has also been used in neurological emergencies such as strokes. "Telestroke" is a model of care, developed as early as 1999 when thrombolysis via injection therapies was developed, where stroke specialists at metropolitan hospitals used telemedicine to remotely diagnose and manage strokes in rural and community hospitals (8). Given the narrow time window that intravenous thrombolysis must be administered within, telestroke avoids time wasted in transferring critical patients to larger hospitals for management.

\section{Wearable/surface sensory technologies}

It has been identified that in various subspecialties that continuous data monitoring provides significant benefit to both patient and provider. Various subspecialties include: cardiology with the use of Holter monitoring (9), continuous blood glucose monitoring for type 1 diabetes (10) and activity monitoring after spinal surgery (11). Figure 1 lists a number of health care metrics that would benefit from continuous monitoring and the relevant pathology that would benefit from such data.

\section{Application of telemedicine and wearable technologies}

\section{Telemedicine}

A standard telehealth consultation should aim to simulate face-to-face communication as much as possible, e.g., by using the video call capabilities of a modern smartphone, tablet, laptop or desktop. Along with being able to inspect the patient, video calls can allow the doctor to inspect the home setting if appropriate, e.g., if there are hazards at home that may require modification.

Compared to other specialities such as radiology, cardiology and psychiatry, the uptake of telemedicine in neurology and orthopaedics has been slow (12). Consultations that do not require physically manipulating the patient may be more amenable to telemedicine consultations (12), and may include monitoring chronic conditions or postoperative patients. However, a face-toface neurological examination is strongly recommended, particularly for the first visit, so that the neurologist can collect appropriate clinical information to make a diagnosis. The standard neurological examination is difficult to translate into a "telehealth at home" setting because patients may not have the required equipment (e.g., tendon hammers). Findings can easily be confounded by poor examination, e.g., conscious suppression of spinal reflexes by higher centres of the central nervous system (13).

To address this issue, there are two potential solutions. Even in the COVID-19 context, patients may still present to emergency departments with acute neurological dysfunction, where the initial assessment is usually performed by a junior doctor. A junior doctor can perform a neurological examination, which is then broadcasted live to a consultant. This allows the consultant to view a competent physical examination, while minimising face-to-face contact and risk of spreading infection. Telemedicine neurological examinations in this format have been shown to be non-inferior to a face-to- 
face examination performed by a junior doctor (14).

For non-urgent consultations where the patient can remain at home, wearable devices can be used instead to act as a surrogate for a formal neurological examination. The concept of wearables has increased in importance, as these devices can detect abnormalities with walking capacity and vital signs etc. $(4,15)$. In addition, various subspecialties could develop a "routine" for video assessment of the patient for a summarised neurological or orthopaedic examination.

\section{Wearable/surface sensory technologies}

Wearable sensory technology allows remote monitoring of many patient metrics by using a small wearable device. These feed data to a database (locally on a phone, or to the cloud) that can be accessed by the patient and physician (16). Many health metrics can be monitored, with vital signs (heart rate, blood pressure, respiratory rate, oxygen saturation, body temperature and electrocardiograms) and cardiovascular function being the focus of many wearable devices (17). From a neurological perspective, the key applications of wearable technologies are gait assessment and postural analysis of patients with acute and chronic spinal pathologies. Applications in the future where wearable sensors could be deployed include: biofeedback training for Parkinson's disease, analysing postural sway in multiple sclerosis, and monitoring recovery after a stroke (5).

Compared to other metrics, gait monitoring with wearable sensors is still in its infancy, as gait is heterogeneous and multifaceted. Sensors in commercial devices such as smartwatches can measure basic metrics such as step count and velocity, however these have a higher degree of inaccuracy, and are not suitable for medical grade monitoring (18).

Wearable devices may have the benefit of more accurately monitoring a patient's gait and posture in a "daily life" setting, which may not be reflected in a controlled test performed in front of a physician. However, if not used correctly, variations in daily movement can generate aberrant findings that may be confusing for the physician.

\section{Barriers with wearables \& telemedicine}

A number of barriers need to be overcome before wearable technology can be reliably used in telemedicine. For example, whilst wearable devices are available for gait analysis, there is no clearly defined gold standard that is both clinically viable and accurate. Inertial measurement units (IMUs) are designed to capture any type of movement in a threedimensional space, and are the go-to sensor for wearable gait analysis devices (15). IMUs usually contain a tri-axial accelerometer to measure linear acceleration in the forward (anteroposterior), sideways (mediolateral) and vertical axes, giving 3 degrees of freedom (DoF) in measurements. However, notwithstanding technological advancement, any such alteration to an IMU to increase measurement accuracy will substantially increase power consumption (19). As such, a compromise must be made to device accuracy to ensure longevity beyond a few hours, which is necessary for remote healthcare. A similar compromise must be made when choosing between single point and multiple point placement of sensors for gait analysis and other health data capture. Sensor placement at multiple points on the body and subsequent data integration allows for detection of more nuanced movements, thorough symmetry analysis and increased sensitivity across all measurements (20). However, multi-point sensors (as in $>3$ DoF IMUs) are more difficult to apply and maintain, especially if they require charging. This inhibits their clinical viability, especially when used for remote monitoring and essentially renders them useless in comparison to less accurate, but far more user-friendly single-point IMUs.

Moreover, the viability of wearable sensors in some scenarios is precluded by the current understanding of the health metric that they are measuring. In elderly populations it is known that certain gait parameters are associated with falls, such as slower gait velocity and cadence (21). However, when similar studies are conducted into patients with neurological gait disorders such as Parkinson's disease and cerebral palsy, the gait patterns of fallers are more nuanced and disease-specific (22). A simple example of this is the monitoring of vital signs (respiratory rate and heart rate) in a recent ex-smoker with coronary artery disease. While a sensory may detect signs in keeping with acute cardiac ischemia, they may also be in keeping with a panic attack due to nicotine withdrawal, COPD-associated tachycardia or exercise. The wearable device is unable to distinguish between them based on the information it can gather, and would require other metrics, or more information on the patterns of tachycardia and tachypnoea that distinguish the aetiologies, which we currently do not have. In a clinical setting, the decision to investigate further would be made by a clinician, informed by patient history and personal experiences, but this cannot be done by the wearable device alone, and would suggest an in-person or telemedicine consult regardless. 


\section{Regulatory and reimbursement issues}

In Australia, prior to the rapid escalation of COVID-19 in the community and well before hospital resources were stretched, the regulatory authorities (Medicare, Australian Government) provided assistance for a range of healthcare practitioners to conduct video-based consultations, with fair financial compensation. This had an immediate impact on face-to-face consultations assisting with the concern of social isolation.

The COVID-19 crisis stimulated policy makers, regulators and payers to encourage expanded use of remote healthcare. Policy restrictions were loosened or lifted. Many telehealth services are now reimbursed with fewer associated administrative burdens. Physicians, regardless of setting, should consider incorporating telehealth into their practice. All health care practitioners need to rapidly understand current local and federal laws, regulations, coding and reimbursement options and stay informed since laws, regulations and reimbursements may change by the day. For example, providers may now be reimbursed for Medicare patients using traditional coding for telehealth visits regardless of patient location, including the patient's home (23). Telemedicine has the capacity to provide national coverage with providers licensed in any US state may now practice telehealth in other states without additional licensing, if state laws allow this $(24,25)$.

Providers must remain vigilant in order to understand their state's laws and each individual payer's policies including use of modifiers, eligible patient populations, etc. Payments by payers within a state vary based on existing parity laws (25), temporary mandates during the emergency period of COVID-19, contract negotiations, and individual payer policy decisions. Further, these rules and regulations may change frequently requiring practices to re-review their contracted payer websites, contacting the payers directly, and/or reaching out to their state medical or neurological societies.

While COVID-19 did not alter telehealth-related Current Procedural Terminology (CPT), new non-faceto-face service codes were added in 2020 (26,27). Practices can benefit from refocusing efforts and fully understanding the wide spectrum of telehealth codes. Providers may be reimbursed not only for traditional telemedicine, but evaluation and management services based on online work, interprofessional consults, or telephone encounters $(6,27)$. However, practices must diligently review each contracted payer's policies to determine applicable payments.
Developing a telehealth-based fee schedule to understand coding and reimbursement implications across payers may help administration as well as providers.

Workflow and staffing functions need to adapt to telehealth services as well. Staff need to support scheduling. IT needs to integrate electronic medical records with telehealth applications if practical, and ensure providers and patients can connect with one another via the telehealth platform. Billing, prior authorisations, managerial oversight, and additional clinical concerns such as prescribing need to be addressed. Depending on size of one's organisation and staffing capabilities, there may be value in creating rotating teams to manage clinic functions in the office or via telehealth. Student and resident education must also be reconfigured to consider their roles whereby they may still "see" patients via telehealth supervised by the attending to discuss best clinical management.

Providers, administrators, hospitals, and networks will benefit from developing models in telehealth to project the economic impact on their practices and institutions. Depending on the organisation size and structure, they may need access to funds and consider overhead reductions to keep their doors open if reserves dwindle due to diminished reimbursements. Consideration should also include small business loans, lines of credit, or other options. Department leadership should be ready for conversations with their hospital or enterprise leadership about projections and ramifications. A sensitivity analysis should consider the impact of an ongoing crisis that may last weeks to months.

In parallel, as practices build or enhance their telemedicine capabilities, they may benefit from ensuring their model is built on a strong, reliable infrastructure, with the ability to scale, and become embedded within normal operations after the crisis resolves (28).

Over time, leadership will also want to understand each provider's strengths and weaknesses associated with telehealth for ongoing utilisation. Telemedicine is a specific audiovisual communications technology, requiring authentic engagement and rapport, provider and patient placement, room staging, nonverbal communication strategies, appropriate documentation, and time management (29). Not every physician may be comfortable with performing a high volume of telemedicine services due to lack of social interaction with colleagues.

The global pandemic will have a lasting effect on society. If there are any positive consequences to be considered, they could include a stronger sense of community, as well 
as ongoing dissemination and implementation of telehealth services that improve population health, patient care, and lower costs.

\section{Benefits and risks of WearTel}

There are benefits and risks to all stakeholders in the transformation of healthcare delivery away from the traditional clinic setting to remote monitoring and delivery of care.

\section{Benefits}

(I) Significant cost reduction: greater implementation of telemedicine has a significant reduction on healthcare and patient costs. Previous implementations of telemedicine, on a much smaller scale, has demonstrated that healthcare costs are reduced by a combination of preventing unnecessary hospitalisation, clinic visits and use of medical resources, and reducing operational costs of running a clinic (30). Further indirect cost reductions can arise from the reduced traffic through the hospital system, such as reduction of the carbon footprint of healthcare;

(II) Continuous objective monitoring: wearable devices offer the potential for continuous objective monitoring of patients. Continuous stream of data on variables such as gait metrics can provide physicians with a better understanding of a patient's health that was not possible in the controlled, potentially biased clinic setting;

(III) Increased access: moving forward, WearTel may be more convenient for some patients, and allow doctors to reach out to patients that may not have accessed healthcare due to travel or cost limitations. Patients from disadvantaged backgrounds, e.g., rural and remote communities, may find healthcare easier to access moving forward if these new changes continue to be supported.

\section{Risks}

(I) Face to face interactions: face to face consultations have important benefits to patient care that cannot be replicated in a telehealth setting. Physicians can have a better grasp or "feel" of the psychological state of the patient when they are face to face, an extremely important factor in determining the flow and direction of the consultation, as well as managing the doctorpatient relationship. The opportunity for interacting with the family unit is also limited in a remote consultation;

(II) Limitations of wearable devices: while consumer grade devices are cost effective and more accessible for patients, they are not validated and precise enough to be clinically useful. While medical grade wearable devices are able to measure health metrics with greater accuracy, the lack of user-friendliness and convenience compared to commercial devices may reduce their appeal to the patient and limit uptake. Furthermore, ongoing research is needed to determine how data captured by wearable devices translates to patient function. Further research will also be needed to develop the algorithms that can use wearable device data for predictive analysis, determining whether a patient is at risk of an adverse event before it occurs.

\section{Conclusions}

Health care practitioners must begin considering what a "post-COVID-19" world may look like. The impact of telemedicine and remote sensing technologies is unlikely to recede, as the shifting paradigm will leave a permanent imprint on health care with new opportunities of care between provider and patient. In addition to remote health care delivery, and as a result of the increased usage of wearable technologies, individual users will have a wealth of data on their own wellbeing at their fingertips, making the consumer far more empowered and equipped to track and monitor their own health and to some extent, even self-diagnose and self-treat. As social distancing appears to become part of normality for the foreseeable future, WearTel will see the lines blurred between the clinic and home like never before.

The current environment, where non-critical patients are encouraged to stay away from hospital if possible, creates an incentive for wearable technology to be implemented as a method of monitoring the health of patients. Careful selection and accurate measurement of health metrics can be used to monitor and detect disease progression early. The potential for the two arms of WearTel to identify and manage patients, before a significant health event requiring hospitalisation occurs, will help reduce healthcare costs and reduce the burden on hospitals.

If we look further in the future however, information asymmetry will transform all current concepts of health care 
delivery, where the digitally agile patient will have far more personal health data based on their own wearable devices than the health practitioner has access to. With continuous objective health data acquisition and artificial intelligence influencing diagnosis and treatment, the physician may be potentially removed from delivery of health care altogether.

\section{Acknowledgments}

The NSURG research group provided assistance with manuscript production. The Wearables and Gait Assessment Research (WAGAR) Group provided assistance with wearables literature.

Funding: None.

\section{Footnote}

Provenance and Peer Review: This article was a free submission to the journal. The article was sent for external peer review.

Conflicts of Interest: All authors have completed the ICMJE uniform disclosure form (available at http://dx.doi. org/10.21037/atm-20-3678). The authors have no conflicts of interest to declare.

Ethical Statement: The authors are accountable for all aspects of the work in ensuring that questions related to the accuracy or integrity of any part of the work are appropriately investigated and resolved.

Open Access Statement: This is an Open Access article distributed in accordance with the Creative Commons Attribution-NonCommercial-NoDerivs 4.0 International License (CC BY-NC-ND 4.0), which permits the noncommercial replication and distribution of the article with the strict proviso that no changes or edits are made and the original work is properly cited (including links to both the formal publication through the relevant DOI and the license). See: https://creativecommons.org/licenses/by-nc-nd/4.0/.

\section{References}

1. Hollander JE, Carr BG. Virtually Perfect? Telemedicine for Covid-19. N Engl J Med 2020;382:1679-81.

2. Mobbs RJ. Spinal News International. Wearables in spine surgery: Beginnings, research and real-world applications 2017. Available online: https://spinalnewsinternational. com/wearables/

3. Mobbs RJ. Spine Universe. Wearing Your Spine Health on Your Sleeve 2017. Available online: https://www. spineuniverse.com/treatments/emerging/wearing-yourspine-health-your-sleeve

4. Mobbs RJ, Mobbs RR, Choy WJ. Proposed objective scoring algorithm for assessment and intervention recovery following surgery for lumbar spinal stenosis based on relevant gait metrics from wearable devices: the Gait Posture index (GPi). J Spine Surg 2019;5:300-9.

5. Yoong NKM, Perring J, Mobbs RJ. Commercial Postural Devices: A Review. Sensors (Basel) 2019;19:5128.

6. American Medical Association. CPT 2020 Professional Edition 2020.

7. Moffatt JJ, Eley DS. The reported benefits of telehealth for rural Australians. Aust Health Rev 2010;34:276-81.

8. Levine Steven R, Gorman M. "Telestroke". Stroke 1999;30:464-9.

9. Steinberg C, Philippon F, Sanchez M, et al. A Novel Wearable Device for Continuous Ambulatory ECG Recording: Proof of Concept and Assessment of Signal Quality. Biosensors (Basel) 2019;9:17.

10. Health Quality Ontario. Continuous Monitoring of Glucose for Type 1 Diabetes: A Health Technology Assessment. Ont Health Technol Assess Ser 2018;18:1-160.

11. Mobbs RJ, Katsinas CJ, Choy WJ, et al. Objective monitoring of activity and Gait Velocity using wearable accelerometer following lumbar microdiscectomy to detect recurrent disc herniation. J Spine Surg 2018;4:792-7.

12. Timpano F, Bonanno L, Bramanti A, et al. Tele-Health and neurology: what is possible? Neurol Sci 2013;34: 2263-70.

13. Gualtierotti T. Inhibition and excitation in spinal reflex activity. J Physiol 1952;117:401-14.

14. Craig JJ, Mcconville JP, Patterson VH. Neurological examination is possible using telemedicine. J Telemed Telecare 1999;5:177-81.

15. Chakravorty A, Mobbs RJ, Anderson DB, et al. The role of wearable devices and objective gait analysis for the assessment and monitoring of patients with lumbar spinal stenosis: systematic review. BMC Musculoskelet Disord 2019;20:288.

16. Majumder S, Mondal T, Deen MJ. Wearable Sensors for Remote Health Monitoring. Sensors (Basel) 2017;17:130.

17. Dias D, Paulo Silva Cunha J. Wearable Health DevicesVital Sign Monitoring, Systems and Technologies. Sensors (Basel) 2018;18:2414.

18. Bassett DR, Jr., Toth LP, LaMunion SR, et al. Step 
Counting: A Review of Measurement Considerations and Health-Related Applications. Sports Med 2017;47:1303-15.

19. Zhu S, Anderson H, Wang Y. Reducing the power consumption of an IMU-Based gait measurement system. Proceedings of the 13 th Pacific-Rim conference on Advances in Multimedia Information Processing. Singapore: Springer-Verlag, 2012:105-16.

20. Della Croce U, Cereatti A, Mancini M. Gait Parameters Estimated Using Inertial Measurement Units. Handbook of Human Motion. Cham: Springer International Publishing, 2018:245-65.

21. Moreira BS, Sampaio RF, Kirkwood RN. Spatiotemporal gait parameters and recurrent falls in community-dwelling elderly women: a prospective study. Braz J Phys Ther 2015;19:61-9.

22. Buckley C, Alcock L, McArdle R, et al. The Role of Movement Analysis in Diagnosing and Monitoring Neurodegenerative Conditions: Insights from Gait and Postural Control. Brain Sci 2019;9:34.

23. Duffy CO, Boyle LM, Nichols BD. HHS Issues Section 1135 Waiver, and CMS Issues Blanket Waivers of Health Care Laws, in Response to Coronavirus (COVID-19) Emergency: The National Law Review, 2020. Available online: https://www.natlawreview.com/article/hhs-issues-

Cite this article as: Mobbs RJ, Ho D, Choy WJ, Betteridge C, Lin H. COVID-19 is shifting the adoption of wearable monitoring and telemedicine (WearTel) in the delivery of healthcare: opinion piece. Ann Transl Med 2020;8(20):1285. doi: 10.21037/atm-20-3678 section-1135-waiver-and-cms-issues-blanket-waivershealth-care-laws

24. Center for Connected Health Policy. Telehealth Coverage Policies in the Time Of COVID-19: Center for Connected Health Policy 2020. Available online: https://www.cchpca. org/resources/covid-19-telehealth-coverage-policies

25. Lacktman NM, Acosta JN, Levine SJ. 50-State Survey of Telehealth Commercial Payer Statutes. Foley \& Lardner LLP, 2019.

26. American Academy of Neurology. Telemedicine and Remote Care 2020 [updated 10/04/2020. Available online: https://www.aan.com/practice/telemedicine/

27. American Academy of Neurology. Procedure Coding for Telemedicine Visits. 2020. Available online: https:// www.aan.com/siteassets/home-page/tools-and-resources/ practicing-neurologist--administrators/telemedicine-andremote-care/20-telemedicine-cpt-codes-and-descriptors.pdf

28. Duffy S, Lee TH. In-Person Health Care as Option B. N Engl J Med 2018;378:104-6.

29. Roth DE. NCTRC Webinar - Telemedicine: How to do it right! [Webinar]: National Consortium of Telehealth Resource Centers; 2019.

30. Noel HC, Vogel DC, Erdos JJ, et al. Home telehealth reduces healthcare costs. Telemed J E Health 2004;10:170-83. 